\title{
Correlation of Haemoglobin Level and Urine Albumin in Relation to Glycated Haemoglobin in Type 2 Diabetes
}

\author{
Sonti Sulochana, Ashok Viswanath, Siddharth Gautam. A.N and Chitra Srinivasan \\ Dept of Pathology, Saveetha university, Saveetha Medical College, Chennai (India)
}

\begin{abstract}
Background; Diabetes mellitus is a chronic metabolic disorder characterized by hypergycemia and derangement in protein and fat metabolism. The prevalence of diabetes was approximately $2.8 \%$ in 2000 and is estimated to grow to $4.4 \%$ by 2030 worldwide.

The Aim and objective of the present study is to correlate haemoglobin, urine albumin with glycated haemoglobin also called as HbA1c testing.

Methods: This study was conducted on 100 Diabetic patients without hypertension, coronary artery disease \& pregnancy. Hb A1c was estimated in diabetic subjects using random EDTA samples by BIORAD D-10 ion exchange high performance liquid chromatography(HPLC), fasting blood glucose was by Glucose oxidase-peroxidase method and haemoglobin estimation by using automated analyzer sysmex XN 1000, Urine albumin measured with URODIP 10A Reagent strips . The data was analyzed by descriptive analysis.

Result and Conclusion: Result shows that hemoglobin was significantly reduced in diabetic patients with high HbA1c and presence of urine albumin which is highly increased in diabetic patients with high glycated hemoglobin. Patients with poor glycemic control are at risk of developing nephropathy. This condition can be controlled by changes in lifestyle and frequent monitoring.
\end{abstract}

\section{Keywords: Glycated Haemoglobin, Haemoglobin, Urine Albumin, Type 2 Diabetes Mellitus}

\section{Introduction}

Diabetes mellitus is a group of metabolic disorder characterized by the presence of high blood sugar for a prolonged period due to defective insulin production or appropriate and efficient utilization of insulin by cells.

It is one of the disorders which are spreading like epidemic all over the world. Inappropriately managed diabetes cause severe complications and decreases the quality of life. The disease and its complications cause a heavy economic burden on patients, their families and society $(1,2)$. The chronic, long term complications are related to damage caused by high blood sugar mainly the blood vessels and are generally classified into micro vascular disease such as diabetic retinopathy (eyes), nephropathy (kidneys), and neuropathy (nerves) and macro vascular disease concerning the heart and blood vessels (large vessel disease). In India the prevalence of retinopathy is high in type 2 diabetic patients and prevalence of nephropathy was less $(8.9 \%$ in Vellore), and 5.5\% in Chennai (3).

Diabetes mellitus is an essential disease characterized by either deficiency of insulin or its peripheral resistance resulting in hyperglycaemic state and nonenzymaticglycation of protein(4). Although poor glycemic control has been associated with cardiovascular disease, nephropathy and retinopathy. Cardio vascular diseases are the primary cause of mortality and morbidity in Type 2 DM patients; and other risk factors, including smoking, hypertension, and dyslipidemia, have been shown to accelerate the progression cardiovascular events(5).Furthermore, elevated urinary albumin excretion is associated with increased risk of Cardio vascular disease(6). Diabetic nephropathy is one of the most common complications, and an important cause of renal failure(7). Measurement of urine albumin should be used for screening, monitoring and to supplement the clinical evidence in the diagnosis and treatment of microalbuminuria.Urinary albumin excretion may represent common pathways for the development of both small and large vessel disease making microalbuminuria as a possible marker for cardiovascular diseases. Micro albuminuria is the earliest clinically detectable stage of diabetes induced damage to kidneys at which appropriate interventions can slow the progression, or even reverse, the process nephropathy(8). Microalbuminuria is defined as urinary albumin excretion rate of $20-200 \mu \mathrm{g} / \mathrm{min}$ or urinary protein excretion rate of $30-300 \mu \mathrm{g} / \mathrm{min}$ predict the future development of nephropathy(9). The risk factors for Microalbuminuria are raised blood pressure and poor glycemic control. Glycosylated haemoglobin is a blood glucose control marker in diabetic patients. HbA1C is formed by condensation of glucose with free amino groups of the globulin component of the haemoglobin 
and normally it comprises $4-6 \%$ of the total haemoglobin and it represents the average glucose concentration over the period of 2-3months. Glycosylated haemoglobin is a useful index of mean glycaemia during the preceding 120 days (10).An increase in blood glucose concentration increases the glycated haemoglobin fraction. It is also a predictor of complications as measures reducing $\mathrm{HbAlc}$ correspondingly reduce the risk of complications (11).

\section{Materials and Methods}

The study was conducted on 100 diabetic patients ( Type-2) , both males $(\mathrm{n}=46)$ and female $(\mathrm{n}=54)$ between age groups of 30-85 years who were registered at Saveetha Medical College and Hospital Saveetha university, Chennai, India. Informed consent of the patient was taken. All patients who were diagnosed diabetes mellitus of type -2 using the ADA criteria of fasting blood glucose (FBG) of $>126 \mathrm{mg} /$ $\mathrm{dl}$ were included in the study. The patients with any recent critical illness were excluded from the study. The blood samples were collected in non vacuum tubes with EDTA as anticoagulant and analyzed within 2 hours of venepuncture for haemoglobin and $\mathrm{HbAlc}$.

HbAlc (glycated haemoglobin) is a form of haemoglobin that is measured primarily to identify the average plasma glucose concentration over prolonged periods of time. Measurement of $\mathrm{HbAlc}$ is used to determine average glycemic control over an 8-12 week period as the life span of the RBcs is $80-120$ days(12). It can be performed at any time of the day and does not require any special preparation such as fasting. More recently, HbAlc test using as a diagnostic test for diabetes and as a screening test for persons at high of diabetes. (13). HbAlc may be affected by a variety of genetic, hematologic and illness related factors (14). The most common important factors affecting HbA1c worldwide are hemoglobinopathies, certain anemias, and disorders associated with accelerated red cell turnover such as malaria (15).

The glycohemoglobin (HbA1c) is formed in two steps by the non enzymatic glycation of HbA.The first step is the formation of an unstable aldimine(labile A1c, or pre A1c), a reversible reaction between the carbonyl group of glucose and the $\mathrm{N}$ terminal valine of $\beta$-chain of haemoglobin.Labile A1c formation is directly propotional to the blood glucose concentration. During red blood cell circulation, some of the labile A1c is converted (Amadori rearrangement) to from a stable ketoamine, $\mathrm{HbAlc}$.

\section{Principles of the Procedure}

The D-10 Hemoglobin A1c program utilizes principles of ion-exchange high-performance liquid chromatography (HPLC). The samples are automatically diluted on the D-10 and injected into the analytical cartridge. The $\mathrm{D}-10$ delivers a programmed buffer gradient of increasing ionic strength to the cartridge, where the hemoglobins are separated based on their ionic interactions with the cartridge material. The separated hemoglobins then pass through the flow cell of the filter photometer, where changes in the absorbance at $415 \mathrm{~nm}$ are measured. Hemoglobin A1 refers to combination of A1a,A1b, and A1c.

Samples for fasting blood glucose estimation was collected in non vacuum fluoride tubes and is estimated by glucose oxidase-Peroxidase method. Haemoglobin is estimated using SYSMEX XN 1000 Automated analyzer .Urine samples were analyzed for albumin using URO-DIP 10 A Reagent strips. The values were obtained and charted in Microsoft excel and descriptively analyzed. ( Fig 1 and Fig 2) .

\section{Analysis}

The data were statistically analyzed by descriptive analysis.

\section{Result}

Among the one hundred diabetic patients with the glycated haemoglobin level more than 6.0 and less than 13.5 with 36 out of 54 female patients showing haemoglobin value of less than 12.0 and 30 out of 46 male patients showing haemoglobin value of less than 13.0. Furthermore among this 66 patients with low haemoglobin value, about 54 patients showed urine protein value of more than $30 \mathrm{mg} /$ dl $(1+)$ with maximum of more than $300 \mathrm{mg} / \mathrm{dl}(4+)$ tested using urine dipstick test(urodip $10 \mathrm{~A}$ strips ). Out of this 66 patients 18 patients showed $30 \mathrm{mg} / \mathrm{dl}(1+)$ proteinuria, 28 patients showed $100 \mathrm{mg} / \mathrm{dl}(2+)$ proteinuria , 11 patients showed $300 \mathrm{mg} / \mathrm{dl}(3+)$ proteinuria 9 patients showed more than $300 \mathrm{mg} / \mathrm{dl}(4+)$ proteinuria, while few of the 46 patients with normal hb value with high glycated haemoglobin level and high fasting blood sugar level showed 5-20mg/ $\mathrm{dl}$ (trace) to $30 \mathrm{mg} / \mathrm{dl}(1+)$ proteinuria.

\section{Discussion}

Type 2 diabetes is the commonest form of diabetes constituting $90 \%$ of the diabetic population in any country. In India, china and U.S more diabetic patients are in the age group of 45-64yrs and more than $65 \mathrm{yrs}$ in the developed countries. Epidemiological studies showed that the prevalence and increased incidence of diabetes was high in urban India (16-18). This is mainly due to life style changes and genetic predisposition in Indian population. In the year 2000 a national survey of diabetes was conducted in six major cities in India showed that the prevalence of diabetes in urban adults was $12.1 \%$ and the prevalence of impaired glucose tolerance was also high (14.0\%). Several studies noted that in Asian Indians the onset of diabetes was at younger age ${ }^{(19,20)}$. 


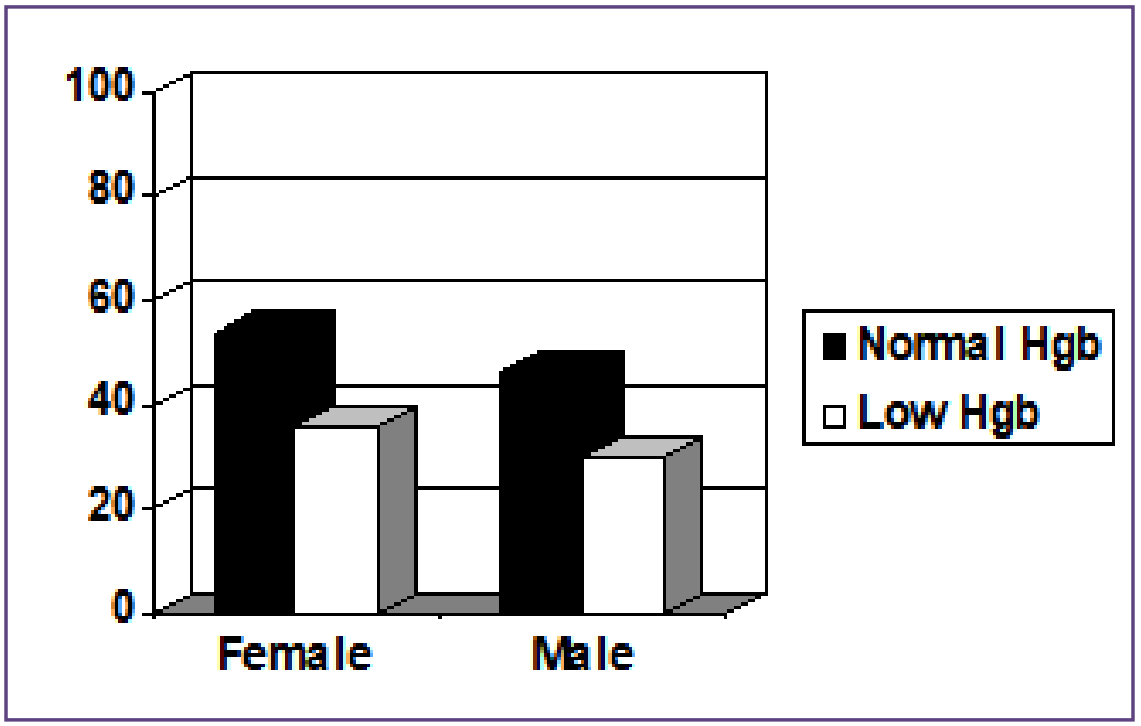

Fig.1: Correlation of Haemoglobin with high Glycated haemoglobin.

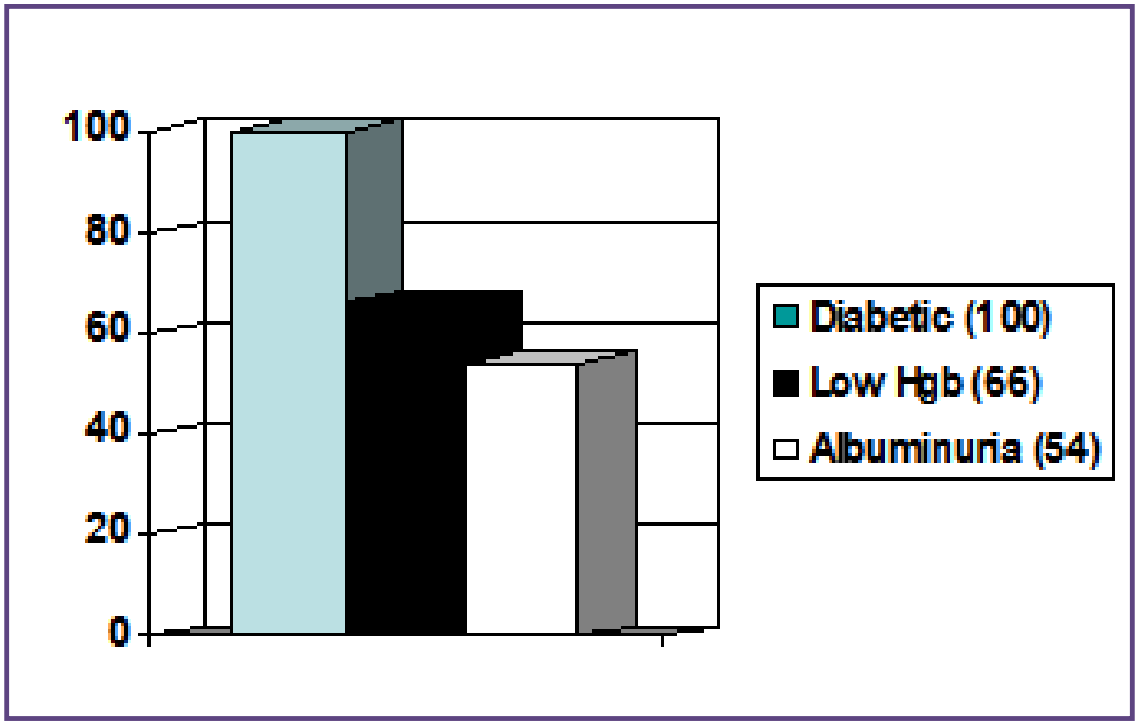

Fig. 2: Correlation of haemoglobin \& urine albumin with high Glycated haemoglobin.

Even though more advances in the healthcare of patients, diabetes remains one of the most common cause of end stage renal diseases ${ }^{(21-25)}$

Hyperglycemia induced variation in haematological parameters and urine albumin has been reported by several studies. Elevation in glucose concentration is one of the major factor that effects erythrocyte morphology i.e. the severity in the change of erythrocyte shape depend upon plasma glucose level. This in turn, affect their flow properties through alteration and deformation at individual level and aggregation at collective level (Singh and Shin, 2009). There is frequently a reduction or change in blood viscosity which predisposes a pearson's system in reacting inadequately to insulin. Significant reduction in haemoglobin was observed in both male and female diabetic patients. Among the 100 diabetic patients, 66 patients showed less haemoglobin value and 54 patients showed presence of urine albumin of varying degree. The reduction in haemoglobin may be due to one among the many complication of diabetes mellitus - diabetic nephropathy, affecting the kidneys resulting in decreased production of erythropoietin resulting in low haemoglobin level.

The presence of urine albumin in normal individuals varies from absence to trace amount of urine albumin, 
which may increase in several conditions with one of the several conditions being diabetes mellitus due to diabetes induced damages to the endothelium of the kidneys leading to leakage of albumin from the blood into the urine.Trans glomerular passage of albumin and tubular reabsorption are important factors plays a role in urinary albumin excretion. Few changes like increased intra-glomerular pressure, loss of negatively charged glycosaminoglycans in the basement membrane and increased basement membrane size, all contribute to the albuminuria. The glomerular and tubular proteinuria can be distinguished simultaneously by measuring the urinary $\beta 2$-microglobulin and albumin. Microalbuminuria does not directly cause cardiovascular events. It is a maker for those who may be at increased risk. It is caused by glomerular capillary injury and so may be marker for diffuse endothelial dysfunction. According to steno hypothesis, albuminuria might reflect a general vascular dysfunction and leakage of albumin and other plasma macromolecules such as low density lipoproteins in to the vessel wall that may lead to inflammatory responses and in turn start the atherosclerotic process $(26,27$. Recently some studies suggested that microalbuminuria may be a risk factor for the development of cardiovascular disease in non diabetics and so it have a role in screening programs (28) The amount of urine albumin increased with increase in level of glycated haemoglobin (HbA1c) indicating the uncontrolled diabetes leading to increased damage to the kidneys. Our results indicate that any change in biochemical parameters particularly glycemic control is reflected by haematological indices particularly haemoglobin which can be used as one of the indication for diabetic complications. There is a need to develop risk factor modification to reduce the impact of long term complications (Khuwaja et al, 2004) Drug therapy such as ACE Inhibitors such as lisinopril along with control of glycemic index is an effective strategy to attenuate the damage occurring to kidney in patients with uncontrolled diabetes for better prognosis and delaying the progression to chronic kidney disease.

\section{Conclusion}

Poor glycemic control causes an alteration in level of haemoglobin in the blood and excretion of varying amount of albumin in urine. Hyperglycemia induces damage to the cells of the kidneys which in long term leads to failure of kidneys resulting in end stage chronic renal disease in diabetics. The study indicates the clinical usefulness of HbAlc, haemoglobin, Urine albumin detection and estimation of the amount as markers for diagnostic point of view and is a cost effective \& very beneficial for patients at high risk of developing micro / macro vascular complications and can help in preventive and early treatment . A Good glycemic control with life style modifications / diet / oral hypoglycaemic drugs / insulin could be supportive and beneficial in reducing the effect of diabetes

\section{Acknowledgement}

We thanks to our colleques Dr.Surekha, Dr. Mahesh Kumar and clinical pathology and biochemistry lab technicians and Medical officer Dr Yogalakshmi and Sabari.

\section{Reference}

1. Ramachandan A,Snehalatha C. Current scenario of diabetes in India. J diabetes 2009;1(1):18 28

2. American Diabetes Association. Diagnosis and Classification of Diabetes Mellitus. Diabetes care 2011 ; 34(suppl 1): 62-69.

3. Ramachandan A,Snehalatha C, Satyavani K, Latha E, Sasikala R, Vijay V. Prevalence of vascular complications and their risk factors in type 2 diabetes. J Assoc Phy India 1999; 47:1152-1156.

4. Harsh Mohan.Endocine pancreas,Diabetes mellitus. Text Book of Pathology. 5th ed. India,Jaypee Brothers Medical Publishers (P) Ltd; 2010: 821

5. Isomaa B, Almgren P, Tuomi T, Forsén B, Lahti K, Nissén M, et al. Cardiovascular morbidity and mortality associated with the metabolic syndrome. Diabetes Care. 2001;24:683-689.

6. Dinneen SF, Gerstein HC. The association of microalbuminuria and mortality in non-insulin-dependent diabetes mellitus. A systematic overview of the literature. Arch Intern Med. 1997;157:1413-1418.

7. Dabla PK. Renal function in diabetic nephropathy. WJD. 2010 ; 1(2): 48-56.

8. Bhat RM, Ganaraja B. A study of renal function tests in South Indian diabetic population. Journal of Pharmacy Research. 2011; 4(12): 4470-72

9. Bruno G, Merletti F, Biggeri A, Bargero G, Ferrero S, Pagano $\mathrm{G}, \mathrm{e}$ al. Progression to overt nephropathy in type-2diabetes. The Casale Monferrato study. Diabetes care 2003;26:21502155.

10. Sacks DB, Brun s DE, Goldstein DE, Maclar en NK and McDonald JM. Guidelines and recommendations for laboratory analysis in the diagnosis and management of diabetes mellitus. ClinChem 2002; 48(3): 436-72.

11. Jha P, Das BKL, Shreshtha S, Majhi S, Chandra L. SharmaS et al. Glycaemic status, lipid profile and proteinuria in diabetic nephropathy. JNepal Med Assoc 2012; 49(178):43-6 .

12. Nathan DM, Turgeon H, Regan S. Relationship between glycated haemoglobin levels and mean glucose levels overtime. Diabetologia 2007; 50: 2239-2244.

13. International expert committee report on the role of the glycated haemoglobin assay in the diagnosis of diabetes. Diabetes care 2009; 32:1327-1334.

14. Gallagher EJ, Bloomgarden ZT, Le Roith D. Review of glycated haemoglobin in the management of diabetes. Journal of Diabetes 2009; 1:9-17. 
15. Roberts WL, De BK, Brown D Et al. Effects of haemoglobin $\mathrm{C}$ and $\mathrm{s}$ traits o eight glycol haemoglobin methods. Clin Chem 2002; 48:383-385.

16. Ramachandan A,Snehalatha C, daisy Dharmaraj, Viswanathan M. Prevalence of glucose intolerance in Asian Indians. Urban rural difference and significance of upper body adiposity. Diabetes care 1992; 15:1348-1355.

17. Ramachandan A,Snehalatha C, Latha eE, Vijay V, Viswanathan M. Rising Prevalence of NIDDM in urban population in India. Diabetologia 1997; 40:232-237.

18. Ramachandan A,Snehalatha C, Kapur A,Vijay V, Mohan V, Das AK, Rao PV, Yajnik CS, Prasanna KS, Nair JD. For the Diabetes Epidemiology Study Group in India(DESI). High prevalene of diabetes and impaired glucose tolerance in India: National Urban Diabetes Survey. Diabetologia 2001; 44: 1094-1101.

19. Ramaiya KL, Kodali VR, Alberti KGMM. Epidemiology of diabetes in Asians of the Indian Sub continent. Diabetes Metabolism Rev1990; 6: 125-146.

20. Jalal DI, Rivard CJ, Johnson RJ, Maahs DM, McFann $\mathrm{K}$, Rewers $\mathrm{M}$, et al. Serum uric acid predict the development of albuminuria over 6 years in patients with type 1 diabetes: Findings from the Coronary Artery Calcification in Type 1Diabetes study. Nephrol Dial Transplant 2010;25:1865-1869
21. Baradaran A. Lipoprotein (a), type 2 diabetes and nephropathy; the mystery continues. J Nephropathology 2012;1:126-9.

22. Rahimi Z. ACE insertion/deletion (I/D) polymorphism and diabetic nephropathy. J Nephropathology 2012;1:143-151

23. Rouhi H, Ganji F. Effect of N-acetyl cysteine on serum lipoprotein (a) and proteinuria in type 2 diabetic patients. J Nephropathology 2013;1:61-66.

24. Tolouian R, Hernandez GT. Prediction of diabetic nephropathy: The need for a sweet biomarker. J Nephropathology. 2013;2:4-5. [PMC free article] [PubMed]

25. Hovind P, Rossing P, Tarnow L, Johnson RJ, Parving HH. Serum uric acid as a predictor for development of diabetic nephropathy in type 1 diabetes: An inception cohort study. Diabetes 2009; 58:1668-71.

26. Wrone EM, Carnethon MR,Panaliappan LP,et al. Association of dietary protein intake and microalbuminuria in healthy adults: Third National Health and Nutrition Examination Survey. Am J Kid Dis 2003; 41: 580-587

27. Deckert T, Feldt-Rasmussen B, Borch-Johnsen K, et al. Albuminuria reflects widespread vascular damage. The Steno hypothesis. Diabetologia 1989; 32:219-226.

28. Gerstein HC, Mann JEF et al. The validity of random urine specimen albumin measurement as a screening test for diabetic nephropathy. Yonesi Med J 1999; 40: 40-45

*Corresponding author:

Sonti Sulochana, Iyyappanthangal,Chennai-600056,T.N,India

Phone: +919600110836

Email: sulochanamaster@gmail.com

Financial or other Competing Interests: None. 